\title{
A Study on the Development Strategy for the Prevention of Infectious Diseases in China
}

\author{
Li Lanjuan ${ }^{1}$, Zhang Boli ${ }^{2,4}$, Xu Jianguo ${ }^{3}$, Du Weibo ${ }^{1}$, Xiao Yonghong ${ }^{1}$, Ma Weihang ${ }^{1}$, Zhang Jianzhong ${ }^{3}$, Zhang \\ Junhua ${ }^{2}$ \\ 1. State Key Laboratory for Diagnosis and Treatment of Infectious Diseases, the First Affiliated Hospital of Zhejiang University, Hangzhou 310003, \\ China \\ 2. Tianjin University of Traditional Chinese Medicine, Tianjin 300193, China \\ 3. National Institute for Communicable Disease Control and Prevention, Chinese Center for Disease Control and Prevention, Beijing 102206, China \\ 4. China Academy of Chinese Medical Sciences, Beijing 100700, China
}

\begin{abstract}
To promote and preserve social harmony, social stability, and sustainable economic development, it is essential to study the current development strategy for the prevention of infectious diseases in China. It is also necessary to propose a regulatory system for a prevention-and-control research strategy for infectious disease that is adaptable to China's specific national conditions. This paper analyzes the epidemic situation and the prevention and control of infectious diseases in China and systematically expounds the significance of conducting a study on the development strategy for the prevention of infectious diseases. Using international experience as a reference, this paper proposes strategic measures and suggestions for a regulatory system and for prevention-and-control research into infectious diseases in China. This study is based on China's achievements and challenges regarding the current system.
\end{abstract}

Keywords: infectious diseases; regulatory system; prevention-and-control research; strategic suggestion

\section{Introduction}

Infectious diseases are generally caused by the invasion of pathogens (viruses, bacteria, fungi, parasites, etc.) in the human body. Among them, contagious diseases are defined as contagions. Infectious diseases pose a serious threat to human health, national security, and social stability with the characteristics of sudden and population-based dissemination. The prevalence of major infectious diseases will cause serious social and economic losses and even hinder the progress of human society [1]. According to the report of the World Health Organization (WHO), infectious diseases account for more than $25 \%$ of all human causes of death and remain the "killer of mankind" [2]. Improving the system of public health and medical service and strengthening capacity-building for prevention and control of major infectious diseases are important tasks in promoting the reform of China's public health undertakings. Conducting strategic research on infectious diseases is of great significance to master the actual conditions of the infectious diseases subject, talents, scientific research, and prevention and control of infectious diseases in China; to formulate new modes and mechanisms of prevention and control of infectious diseases in line with China's national condition; to promote the reform of medical and health care; to safeguard people's health; and to promote sustainable development of China's society and economy and even achieve the "Chinese Dream."

\section{Received date: November 30, 2016; Revised date: December 20, 2016}

Corresponding author: Li Lanjuan, State Key Laboratory for Diagnosis and Treatment of Infectious Diseases, the First Affiliated Hospital of Zhejiang University, Chairman; Chinese Academy of Engineering, Academician. Major research fields include diagnosis and treatment of infectious diseases, liver failure and artificial liver, and infectious microecology. E-mail: 1jli@zju.edu.cn

Funding program: CAE Advisory Project “A Study on the Development Strategy for the Prevention of Infectious Diseases in China” (2012-XZ-08)

Chinese version: Strategic Study of CAE 2017, 19 (2): 037-042

Cited item: Li Lanjuan et al. A Study on the Development Strategy for the Prevention of Infectious Diseases in China. Strategic Study of CAE, https://doi.org/10.15302/ J-SSCAE-2017.02.005 


\section{Significance of research on the current situation and prevention-and-control strategy for infectious diseases in China}

\subsection{The prevalence of infectious diseases is not optimistic in China}

At present, human immunodeficiency virus (HIV) infection is growing rapidly and invisibly in China. The incidence of acquired immunodeficiency syndrome (AIDS) shows an upward trend; as of 2014, the number of people infected with HIV in China has exceeded 850 000; and the epidemic of HIV infection has been severe in some particular areas [3]. A national epidemiological survey of chronic hepatitis B revealed nearly 100 million hepatitis B virus carriers and chronic hepatitis patients in China. The social burden of viral hepatitis and related diseases is still heavy because the problem of hepatitis becoming chronic and severe is prominent, and the prognosis is poor. The number of tuberculosis cases exceeds one million in China and the diagnosis and treatment of drug-resistant tuberculosis is very difficult. The number of multidrug-resistant tuberculosis (MDR-TB) cases ranks among the highest in the world, and the tuberculosis epidemic rate remains high [4]. On the other hand, new and unexpected infectious diseases continue to emerge, including severe acute respiratory syndrome (SARS), human-bird flu, hand-foot-mouth disease, Streptococcus suis infection, and fever with thrombocytopenia syndrome, and threaten societal and economic development. Public health problems such as nosocomial infections, opportunistic infections, and drug-resistant infections are also getting severer [5] According to data from the China Antimicrobial Resistance Surveillance System (CARSS) in 2011, the national clinical bacterial resistance was serious in China with bacteria in Enterobacteriaceae producing extended-spectrum $\beta$-lactamase, and multi-drugresistant non-fermentative bacteria were very common, increasing the difficulty of infection control [6].

\subsection{The situation of prevention and treatment of infectious diseases is severe in China}

The prevention and control of infectious diseases in China are under double pressure from traditional and new infectious diseases. Since the 21st century, domestic outbreaks of new infectious diseases have been frequent, and the threat of traditional infectious diseases has been constant. Many new infectious diseases are acute, and, due to difficult early detection and diagnosis as well as the lack of specific means of prevention and control, early mortality is high. The progress of urbanization has increased population mobility; thus, the vaccination of infectious diseases is difficult to implement, and prevention and treatment of infectious diseases are more difficult [7]. The particulars of infectious diseases such as acute onset, lack of specificity, and difficult early diagnosis contribute to the increasing seriousness of the situation of infectious diseases in China. The risk of international infectious diseases is 002 also escalating. Most cases of malaria in China are imported from overseas. Unsafe sex leads to high incidence of AIDS and syphilis. Poor sanitation conditions in some parts of the economically underdeveloped rural areas have caused some zoonoses to continue threatening the health of local populations.

2.3 The significance of carrying out research on the development strategy of infectious diseases

Effective prevention and treatment of infectious diseases has always been the focus of international attention and is also the key to promoting the social and economic development of the region and the country. China has included the prevention and treatment of major infectious diseases in the Outline of the National Plan for Medium- and Long-term Scientific and Technological Development (2006-2020), which has put forward the national strategic goal that " $[\mathrm{t}]$ he level of prevention and treatment of major diseases would be increased significantly, [and] AIDS, viral hepatitis and other major diseases could be suppressed effectively after 15 years of hard work." At present, the economic level has steadily improved, the people's material standard of living has improved overall, and the disease preventionand-control system has improved daily in China. Significant progress has been made in various aspects such as diagnosis, prevention, and treatment of major and new-burst infectious diseases. However, compared with Western developed countries, some weak links remain in the prevention and control of infectious diseases, and the long-term working mechanism must be further improved. At present, it is very urgent to conduct strategic research on the prevention and control of infectious diseases in China. The main problems and shortcomings in the diagnosis, prevention, and treatment of infectious diseases in China and in Western countries must be compared and analyzed from the perspectives of scientific research, clinical diagnosis and treatment, platform construction, personnel training, and construction of specialist teams. Then, a new model and new mechanism must be presented suitable for the national conditions of China and for research on the development strategy of prevention and control of infectious disease to promote the reform of medical and health care, safeguard people's physical and mental health, and promote social harmony and stability and sustainable economic development in China.

\section{The international experience and inspiration of system construction and the research of prevention and control on infectious diseases}

\subsection{The international experience of infectious disease discipline} system construction and prevention-and-control research

3.1.1 Scientific research needs collaborative innovation and extensive cooperation

Collaborative innovation is the development trend of inter- 
national scientific research of infectious disease. The research and management of infectious diseases in Europe and the United States are still leading the world. Most of the pathogens of various infectious diseases were discovered by these countries, and various therapeutic medicines for infectious diseases and preventive vaccines come from them. These research achievements benefited from their good team of personnel, collaborating mechanisms, and research facilities, such as National Institutes of Allergy and Infectious Diseases, Institut Pasteur in France. There are boundless examples of extensive international collaborative innovation in infectious diseases that have made great achievements, such as the discovery of the SARS virus in 2003, the research on the bird flu virus in 2009, and the identification of the source of Enterohemorrhagic Escherichia coli in Europe in 2011. These collaborations span not only the boundaries of countries and regions but also the wisdom of scientists from major research institutions worldwide and are the results of longterm collaborative research [8].

\subsubsection{Cultivation orientation, fostering top-notch talents}

In recent years, great changes have taken place in the international model of medical education. With economic globalization and the rapid development of medical science and technology objectively putting forward higher requirements for medical personnel training, medical education will no longer be simply oriented toward teaching and imparting knowledge [9]. The standard concept and developmental trend of international medical education have gone from the traditional Flexner course model in 1911 to the spices model course proposed by Harden in 1984 -namely, student-centered and problem-based, integrated, community-oriented, elective, and systematic - and will gradually transition to competency-based programs. Thanks to tremendous investments in medical research and the success of medical education, medical research has made great achievements in the United States over the past century, and many medical Nobel Prize winners have emerged.

The reform of the medical curriculum model in Western developed countries has brought major changes in the training objectives of medical students and is expected to cultivate leading talents in relevant disciplines and fields of specialization. Infectious disease is an important clinical practice course. Students should have not only a solid basic knowledge of infectious diseases, strong clinical practice skills, and systematic group health knowledge but also good professional ethics and correct professional values, broader knowledge of humanities and social science, strong interpersonal communication, information management and lifelong learning ability, and so on.

\subsubsection{Improve the system of specialist training}

In the United States, medical students must learn 12 years of basic and medical knowledge to become qualified physicians. To become specialist students, they must go through a period of university education, medical school education, post-graduate training, and professional training. Since entering the 21 st century, after being qualified as a United States specialist, specialists must undergo qualification recertification every 7 to 10 years. Physicians must continuously receive medical education, take exams, and conduct qualification examinations. The regular examination system for qualifications of specialists strengthens the quality of continuing education of doctors and guarantees the professional level of physicians. Compared with the domestic medical education system, medical students in developed countries such as Europe and the United States have received more systematic, comprehensive, and standardized education and training. At the same time, the access and management system of physicians is also almost perfect. School education and post-graduation education link up well; theoretical teaching and practical training are comprehensive and complete. Systematic training system guarantees the training quality and the professional and technical level of specialist and general practitioners [10].

\subsection{Inspiration for the construction and development of a discipline system in infectious diseases in China}

3.2.1 Scientific research must be collaborative and innovative

Collaborative innovation is conducted to overcome major scientific problems of infectious diseases, creating a world-class cluster of disciplines leading the research direction of infectious diseases [11]. The occurrence of infectious diseases must have the three basic elements of pathogens, transmission routes, and susceptible populations. The diagnosis-and-treatment research on infectious disease requires multidisciplinary collaboration to be clear of the pathogen, establish the pathogenesis, master and cut off the transmission, and develop effective treatment of drugs and vaccines. The relevant universities, medical institutions, research institutes, and disease control departments must work in close coordination and integration of superior forces to rapidly form a co-innovation team with unified goals and doubled efficiency and carry out research combining bench-side work with the bedside as well as prevention and treatment to control the outbreak of infectious diseases in the shortest possible time and minimize its harm.

3.2.2 To intensify the role of infectious diseases disciplines and to increase investment of scientific research in the field of infectious diseases

Most developed countries classify infectious diseases as third-class disciplines; physicians in infectious disease department are more committed to hospital consultation tasks. Infectious disease physicians in the United States are required to participate in the control of nosocomial-acquired infection, diagnosis and treatment of immunocompromised populations and patients with HIV/AIDS and fever, and instruction in the use of antibiotics throughout the hospital [12]. Samsung Medical 
Center in South Korea has set up an infectious diseases ward and outpatient clinic, and infectious disease physicians should be responsible for HIV/AIDS counseling as well as other types of infectious diseases, consultation work on nosocomial infectious disease, and instruction in the use of antibiotics. There is a big gap between China and developed Western countries in terms of investment in science and technology for infectious diseases [13].

3.2.3 To create personnel training mode with global perspective and innovation team

Collaborative innovation is conducted to bring together and cultivate high-end personnel engaged in infectious disease research and build a steady and sustainable population of talents. To improve the ability and level of diagnosis and treatment of infectious diseases in China, we must innovate the personnel training mechanism and establish a personnel training mode with a global concept. We must change the method of appraising talents simply with essay and establish a talent-evaluation system centered on "quality and contribution," actively introduce high-end talents and cultivate reserve talents, and establish a contingent of innovative talents with "well-trained, outstanding capabilities devoted themselves to research and professional enthusiasm" [14].

3.2.4 Improve the training system of specialists and the professional quality of specialists

From the clinician's education and training to the access management, we must establish and improve the specialist/general practitioner system, emphasize the clinician's basic clinical skills training, and improve the quality of clinician's medical service. We should strengthen relevant work on "medicine, teaching and research"; strengthen the training on the rational use of antiinfection drugs; do a good job in teaching and researching new infectious diseases, legal infectious diseases, and various non-contagious infectious diseases; and promote all-around development of disciplines and breakthroughs in innovation. We must further expand the scope of infectious diseases, from the identification, diagnosis, isolation, and treatment of contagious diseases to the diagnosis, treatment, control, and rational application of anti-infective drugs of all kinds of infectious diseases (including contagious diseases) [15].

\section{Achievements and challenges in the research into a discipline system and prevention and control of infectious diseases in China}

\subsection{Achievements in the research of discipline system and prevention and control of infectious diseases in China}

4.1.1 Investments in national science and technology increased, scientific research quality improved, and transformation of scientific and technological achievements accelerated

Over the past decade, China has continuously increased investments in science and technology. Prevention and control of infectious diseases has become a hot research area. Many projects have been funded by the National Natural Science Foundation of China, the "863" Program, the "973" Program, and the National Key Science and Technology Projects. The Outline of the National Plan for Medium- and Long-term Scientific and Technological Development (2006-2020) has established a major project of science and technology on prevention and control of major infectious diseases such as AIDS and viral hepatitis, in which more than 10 billion has been invested, and has achieved remarkable results. It is the most supported project for prevention and control of infectious disease since the foundation of the People's Republic of China.

4.1.2 Medical treatment system of infectious disease was further improved, and the medical treatment capacity increased dramatically

In 2012, the number of registered doctors in infectious disease hospitals increased by $58 \%$, and the number of beds in hospitals of infectious disease increased by $64 \%$ in the past eight years. China gradually established a clinical network of prevention and treatment of infectious diseases with Chinese medicine. In 2013, more than 200 hospitals were identified to construct the clinical base for prevention and treatment of infectious diseases with Chinese medicine, and more than 2000 general hospitals set up infectious diseases divisions. In the outbreak of epidemics, the medical treatment of infectious diseases in China obtained good achievements in 2013. While facing H7N9 bird flu, the whole country worked together to control the spread of the epidemic and ensure public safety and got approval and recognition from the $\mathrm{WHO}$, society, and the masses. At present, the capacity of treatment of communicable diseases in China has risen sharply; relevant medical institutions, emergency rescue centers, and infectious diseases hospitals and other institutions have continuously strengthened; and the medical treatment system for infectious diseases has been further improved.

4.1.3 The construction of national research base and platform has been strengthened, and the construction of a collaborative innovation center of diagnosis and treatment for infectious disease has achieved remarkable results

Since the 11th Five-Year Plan, several key national laboratories, the National Engineering Research Center, and the National Engineering Technology Research Center have been established and developed in the fields of basic clinical and transformational aspects of infectious diseases to ensure improvement in the capability of technological innovation and achievement transformation. The functions of national, provincial, and ministerial scientific research platforms are becoming increasingly perfect and open; the construction of domestic research bases and platforms has been strengthened; and the disciplinary and technological research equipment has been supplemented and improved 
by upgrading, introducing, and independently researching. The Collaborative Innovation Center for Diagnosis and Treatment of Infectious Diseases was established by Zhejiang University and jointly with the advantages of scientific and technological strength and resources such as Tsinghua University, The University of Hong Kong, and Chinese Center for Disease Control and Prevention. It has been identified by the Ministry of Education and Ministry of Finance. This center positioning advanced scientific development with the integration and coordinated development of talent, discipline, and scientific research and plays a key role in prevention and treatment of major infectious diseases and combating new-burst infectious disease.

4.1.4 Multi-leveled and -channeled system of talents introduction and training was built, and the prevention-and-control system of infectious disease was basically established

Based on the overall planning, China has vigorously introduced the urgently needed personnel through various intellectual projects, thus narrowing the gap between China and rest of the world in certain key areas to a certain extent. By actively creating conditions, the introduced personnel can become the leading talents to accomplish arduous tasks and impact the skill-assembling effect, which may generate powerful and technical breakthroughs. At the same time, China has established the largest network of a direct reporting system in the world to conduct whole-network supervision on prevention and control of epidemic situation of communicable diseases and public health emergencies. The ability to cope with major burst epidemics has improved significantly through continuously improved systems including the prevention-and-control system of related disease, the prevention-and-treatment and surveillance system of infectious disease, and the continuously strengthened ability of monitoring and early warning of sudden outbreaks [16].

\subsection{Infectious disease discipline system and the challenges of prevention-and-control research}

\subsubsection{Scientific creativity must be continuously strengthened}

The current system of medical science and technology innovation in China not only lacks intersection and integration between medicine and other disciplines but also presents a clear disassociation between basic and clinical medicine. Many scientific studies are still in the follow-up and accumulation stage, and the source of innovation is not strong. There are more followup studies, and fewer original innovation works. Research is scattered, lacking systematic process, depth, and strength; the scientific, sustainable, and balanced investment still needs to be strengthened. The monitoring and early warning system is not perfect, the large-scale population investigation is insufficient, and the monitoring index system and the core monitoring technology are lacking. The low innovative capacity of diagnosis and treatment and drugs seriously hampers the improvement of national innovation capability and competitiveness and affects national security, and scientific research achievements are not fast-moving, and their proportion is not high. The phenomenon of disconnection among "production, study, research, and use" remains very serious.

\subsubsection{The overall clinical business is limited}

The overall business of infectious diseases discipline is limited, and the incidence of infectious diseases other than viral hepatitis is significantly reduced; clinical departments and subdivisions are increasingly fine, and there is competition among disciplines, mainly because the economic indicators of the hospital performance appraisal system seriously hindered the development of the infectious diseases discipline. The diagnosis and treatment of infectious diseases are mostly lacking high valueadded treatment, which is bound to affect profit and loss and the development of infectious subjects. In some areas, the degree of emphasis on infectious diseases is insufficient, the hospital's infection department is withdrawn, and the phenomenon is serious. The lack of rapid and accurate diagnosis and treatment (especially pathogen examination) has led to a lack of control over various infectious diseases [17].

\subsubsection{Related technical support must be improved}

Scientific research bases, platform construction, and the science and technology soft environment must still be strengthened. Globally, there is still a long way to go from the international first-class scientific research technology in infectious diseases of China. The soft environment of some scientific research institutions is not recognized by their scientific and technical personnel. The conditions for scientific and technological support and the platform for the development of disciplines lack a higher level of planning and coordination; monitoring and early warning system remains imperfect; related technologies and equipment, technology platforms, and standards have not been established; and traditional Chinese medicine has not been given a full role in the prevention and treatment of infectious diseases.

4.2.4 Skill training cannot meet the demand; the basic quality of specialist teams must be improved

Inadequate investment in countries shrinks disciplines or limits development, resulting in some brain drain, and most outstanding graduates are not willing to engage in clinical research and teaching of infectious diseases. For infectious disease teaching content, the teaching model is too old and backward; the teaching content has failed to adjust with changes in the disease spectrum, and teaching content has broken away from clinical practice. Thus, teaching materials urgently needed to be edited. Most of the current teaching materials are Communicable Diseases teaching materials while Infectious Diseases teaching materials are few. Infectious disease curriculum and teaching model are aging, theoretical classes and other disciplines are 
lacking, bedside learning opportunities are too little, and professional theory and clinical practice are out of touch [18]. The existing teaching mode cannot adapt to the needs of personnel training. The teaching content, curriculum, and teaching mode of infectious diseases must be deeply considered and thoroughly changed. At present, the educational qualifications and professional structure of the infectious disease specialist team in China are hard to adapt to the needs of the treatment of infectious diseases. The defects of the original specialist training system result in uneven quality of physicians. At present, the contingent of specialist physicians whose basic quality must be improved cannot meet the needs of the masses, high standards, high levels of infectious disease prevention, and treatment service's needs.

\section{China's infectious disease disciplinary system and its prevention-and-control research measures and suggestions}

\subsection{Enhancing prevention and treatment of infectious diseases to the height of national strategic safety and strengthening top-level design and investment in scientific research in prevention and control of infectious diseases}

We will raise the awareness of prevention and control of infectious diseases to a level related to national security and further strengthen the top-level design, improving the coordination mechanism, implementing a long-term strategy, and establishing a new national prevention-and-control system for infectious diseases. We will develop infectious diseases from the perspective of the "needs of the nation" rather than the "needs of experts," do a good job in the near- and long-term development of a top-level design for prevention and control of infectious diseases, break down the current "top-down" design mode from the bottom up, break down departments with binding interests, and form a national-level think tank decision-making team. In the basic and applied fields, we should invest more in research on medical microecology, bacterial resistance, prevention and control of nosocomial infections, and prevention and treatment of infectious diseases by Chinese medicine and set up special funds for support as soon as possible. Infectious diseases should be China's key disease prevention and control and must continue to be included in the next national long-term development plan. We will further improve the mechanism for financial input and increase investment so that long-term plans for major infectious diseases and technologies will be well targeted considering the outstanding issues at this stage.

\subsection{Construction of an infectious diseases clinical treatment and translation medical center}

It is suggested that a center for clinical treatment and translational medicine should be established as a part of the national emergency capacity building to bring together resources such as research teams and clinical bases for the treatment of infectious diseases in China; to build an advanced platform for scientific research and translation including Level 4 biosafety laboratories; and to develop and rapidly reduce the incidence of serious and severe infectious diseases through the multidisciplinary collaboration of basic medicine and clinical medicine, clinical medicine, and traditional Chinese medicine. We should track the rate and mortality of key technologies, methods, and programs to effectively treat Ebola, hemorrhagic fever, and other potent and new infectious diseases; reduce mortality; and establish strategic and technical reserves.

\subsection{Improve the treatment of infectious diseases medical staff}

Healthcare workers are the dominant force in treating infected patients and reducing the mortality rate and have made tremendous contributions to their effective response to major and new unexpected infectious diseases. Heavy work pressure, high labor intensity, occupational exposure, poor working environment, healthcare workers' income, and social contribution are significantly inconsistent. Infection department healthcare workers' wages and benefits are significantly low, exacerbating the brain drain. Infectious diseases experts lack stability. It is suggested that the government should raise special subsidies for medical staff working with infectious diseases, earnestly raise the income level, and give preferential policies on the introduction and exchange of relevant personnel for infectious diseases and the assessment of professional titles.

\subsection{Increase international cooperation and rescue, layout of clinical research "overseas base"}

We will step up international cooperation and assistance, support and participate in the activities of international organizations, further expand the research space for new and reproductive disease treatment, and help win cooperation while helping aid stricken countries. It is suggested that the "overseas bases" should be laid out to help enhance clinical and scientific emergency capabilities and obtain information resources, bacteria (strains) strains, and sample resources with potential extensions and strategic values to provide important protection for effectively carrying out research on new onset and reproductive diseases in China.

5.5 Improve disciplinary construction, change "communicable diseases" to "infectious diseases," and add "medical microecology" as a first-level discipline

The translation of communicable diseases has been unable to cover the content of infectious diseases, which is very un- 
favorable to discipline development and personnel training. Medical microecology has become the hotspot and forefront of international scientific research. Western developed countries make key deployment in the fields of human microecological structure analysis, microecological imbalance and health, microecology, and biological safety. It is suggested that the Office of the State Council Academic Degrees Committee should adjust its disciplinary classification and change its name to "infectious diseases" and be classified as a first-level discipline with the addition of "medical microbiology" (a first-class subject) and, with the change of discipline classification, that the corresponding textbook be changed to Infectious Diseases and the corresponding clinical department changed to "Infectious Diseases." We will continue to increase investment in science and technology in the field of microecology and set up special microecological research projects in the field of the National Natural Science Foundation of China, National Major Research Programs, and National Applied Research Programs to promote the continuous production of original scientific research results and maintain the role of an international leader.

\section{References}

[1] Li L J. Status and control strategy for infectious diseases in China [J]. Chinese Journal of Clinical Infectious Diseases, 2008, 1(1): 1-6. Chinese.

[2] World Health Organization. The top 10 cause of death [R]. Geneva: World Health Organization, 2014.

[3] Fan X M. Status and strategy for AIDs control in China [J]. Truth Seeking, 2014 (A01): 190-192. Chinese.

[4] World Health Organization. Antimicrobial resistance: Global report on surveillance [R]. Geneva: World Health Organization, 2014.

[5] Center for Disease Control. Antibiotic resistance threat in US [R] Altlantic: Center for Disease Control, 2013.

[6] Xiao Y H, Shen P, Wei Z Q, et al. Mohnarin report of 2011: Monitoring of bacterial resistance in China [J]. Chinese Journal of Nosocomiology, 2012, 22(22): 4946-4952. Chinese.
[7] Gao H N, Yao H P, Yang S G, et al. From SARS to MERS: Evidences and perspective [J]. Chinese Journal of Clinical Infectious Diseases, 2015, 8(4): 292-298. Chinese.

[8] Wang M L, Sun J, Wang Y D, et al. Inspiration for China's management of emergency from American emergency prevention system based on scenarios building [J]. Medical Education Management, 2016, 2(2): 458-463. Chinese.

[9] Wen Z Y, Wang Y M, Yang X M, et al. Take international certification as an opportunity in medical education and explore establishment the whole system of quality assessment of biochemistry learning [J]. Basic \& Clinical Medicine, 2015, 35(2): 278-280. Chinese.

[10] Silva C J, Torres D F M. Optimal control for a tuberculosis model with reinfection and post-exposure interventions [J]. Mathematical Biosciences, 2013, 244(2): 154-164.

[11] Laxminarayan R, Sridhar D, Blaser M, et al. Achieving global targets for antimicrobial resistance [J]. Science, 2016, 353(6302): 874-875

[12] Soothill G, Hu Y, Coates A. Can we prevent antimicrobial resistance by using Antimicrobials better? [J]. Pathogens, 2013, 2(2): $422-435$.

[13] World Health Organization. Global tuberculosis control: Epidemiology, strategy, financing [R]. Geneva: World Health Organization, 2009.

[14] Lian J Q, Wang J P, Zhang J C. Innovation and exploration of the mode of infectious disease teaching [J]. Northwest Medical Education, 2009, 17(3): 608-610. Chinese.

[15] Anya I, Ihekweazu C. Democracy in Nigeria: The challenge of infectious disease control [J]. Journal of Infection in Developing Countries, 2008, 2(2): 151-153.

[16] Cao J G. New progresses and development vision of hospital infections [J]. Medical Journal of Chinese People's Liberation Army, 2010, 35(11): 1277-1280. Chinese.

[17] Xiao Y H. Infectious disease physician should be the backbone of antibacterial agent rational use [J]. International Journal of Epidemiology and Infectious Disease, 2009, 36(1): 1-3. Chinese.

[18] Zhang Y, Yu Y S, Tang Z H, et al. Problems and reform measures in current teaching of infectious diseases [J]. Chinese Journal of General Practice, 2015, 13(4): 661-663. Chinese. 